\title{
Research Note \\ On highly eccentric stellar trajectories interacting with a self-gravitating disc in Sgr $A^{\star}$
}

\author{
L. Šubr ${ }^{1}$ and V. Karas ${ }^{2,1}$ \\ ${ }^{1}$ Charles University, Faculty of Mathematics and Physics, V Holešovičkách 2, 18000 Prague, Czech Republic \\ e-mail: vladimir.karas@cuni.cz \\ 2 Astronomical Institute, Academy of Sciences, Boční II, 14131 Prague, Czech Republic
}

Received 29 September 2004 / Accepted 30 December 2004

\begin{abstract}
We propose that Kozai's phenomenon is responsible for the long-term evolution of stellar orbits near a supermassive black hole. We pursue the idea that this process may be driven by a fossil accretion disc in the centre of our Galaxy, causing the gradual orbital decay of stellar trajectories, while setting some stars on highly elliptic orbits. We evolve model orbits that undergo repetitive transitions across the disc over the period of $\approx 10^{7}$ years. We assume that the disc mass is small compared to the central black hole, and its gravitational field comparatively weak, yet non-zero, and we set the present values of orbital parameters of the model star consistent with those reported for the S2 star in Sagittarius A*. We show how a model trajectory decays and circularizes, but at some point the mean eccentricity is substantially increased by Kozai's resonance. In consequence the orbital decay of highly eccentric orbits is accelerated. A combination of an axially symmetric gravitational field and dissipative environment can provide a mechanism explaining the origin of stars on highly eccentric orbits tightly bound to the central black hole. In the context of other S-stars, we can conclude that an acceptable mass of the disc (i.e., $M_{\mathrm{d}} \lesssim 1$ per cent of the black hole mass) is compatible with their surprisingly young age and small pericentre distances, provided these stars were formed at $r \lesssim 10^{5}$ gravitational radii.
\end{abstract}

Key words. Galaxy: centre - accretion, accretion-discks - black hole physics

\section{Introduction}

A dense nuclear cluster surrounds the centre of our Galaxy. Tracking the rapid proper motion of individual stars in the central arcsecond (i.e., about $0.04 \mathrm{pc}$ off $\mathrm{Sgr} \mathrm{A}^{*}$ object) provides an essential tool to determine the central mass $M_{\bullet} \approx 3.5 \times 10^{6} M_{\odot}$ (Genzel et al. 2003; Ghez et al. 2003a). So far, the motion of S2 (S0-2) has been measured with the best accuracy over a sufficient duration of time (Schödel et al. 2002, 2003). It is a highly eccentric, $e=0.87$ Keplerian orbit with the orbital period of 15.2 years and an apocentre distance $0.01 \mathrm{pc}$. Other newly discovered stars strengthen the case for a supermassive black hole in $\mathrm{Sgr} \mathrm{A}^{*}$, and for the surprisingly young stellar population around it. The rather high mass and low age present a challenge to star-formation theories (Milosavljević \& Loeb 2004; Eckart et al. 2004). In this paper we discuss one of the possible mechanisms that could help to resolve the issue.

$\mathrm{S} 2$ is a member of the central Galactic cluster for which radial velocity as well as proper motion have been reported by Ghez et al. (2003b). Combination of the two pieces of information allowed these authors to determine the orbital parameters from the full three-dimensional motion and to break the ambiguity in the inclination angle. Trajectories and spectroscopic measurements of other stars in this region have not yet reached comparable accuracy, however, there are good prospects for future progress and strong indications of the puzzling situation: a number of S-stars are on close orbits near the black hole but they seem to be of young age, contrary to expectations. Several mechanisms have been proposed that could bring stars to the neighbourhood of the central black hole (e.g., Hansen \& Milosavljević 2003; McMillan \& Portegies Zwart 2003; Alexander \& Livio 2004). In spite of this effort the origin of close stars is not well understood. Models have difficulties in reconciling different aspects of the Galaxy centre - on the one hand it is a low level of present activity, indicating a very small accretion rate, and on the other hand the spectral classification suggests that these stars have been formed relatively recently. While in-situ star formation is problematic because of strong tidal forces operating near the central black hole, two-body gravitational relaxation could in principle set stars on plunging orbits. Nonetheless, the latter mechanism does not seem to be efficient enough because a rough estimate of the relaxation time-scale, $t_{\mathrm{r}} \gtrsim 1 \mathrm{Gyr}$, is orders of magnitude longer than the estimated age of the S-stars $(\approx 10 \mathrm{Myr})$. We neglect the two-body gravitational scattering and the effect of gravitational relaxation in the present analysis (see Alexander \& Hopman 2003 for a more elaborate discussion of gravitational relaxation in the Galactic Centre). 
The interaction between stars and a fossil accretion disc is a cumulative effect which provides a viable mechanism of transporting stars towards the black hole, while stripping their outer atmosphere away. These two effects are mutually linked and they complement one another: whereas the stellar orbits are evolved by the interaction with the disc, both gravitational and hydrodynamical as discussed in this paper, the stars themselves as well as the composition of the gaseous environment are also affected (Artymowicz et al. 1993; Armitage et al. 1996). The main appeal of this scheme lies in the fact that it naturally combines several influences which are needed in order to explain the efficient migration of stars to the centre: the proposed scenario sets some stars from the sample on elliptic orbits for a fraction of their lifetime (the gravitational effect of the disc), it also causes continuous migration (hydrodynamical effect of the orbital dissipation), and finally it affects stars by removing their atmospheres. All these effects are determined by the assumed density profile of the disc and they are thus interconnected. Furthermore, it was proposed that S-stars have undergone rejuvenation by the interaction with the environment or by tidal squeezing during close encounters with the black hole, thereby hampering the spectral classification and mimicking young age (e.g. Morris 1993; Alexander \& Livio 2001). Notice, however, that we neglect these peculiarities, nor do we take into account the likely effect of the disc itself evolving.

Various authors have investigated the long-term orbital evolution of a stellar satellite that is either periodically crashing on the black-hole accretion disc (e.g., Syer et al. 1991; Vokrouhlický \& Karas 1993, 1998a; Rauch 1995; Šubr \& Karas 1999) or stays embedded in the disc plane (Artymowicz 1993; Hameury et al. 1994; Karas \& Šubr 2001). The abovementioned papers address the star-disc interaction in active galactic nuclei, whose accretion discs are supposed to be rather dense, however, it has been suggested (Levin \& Beloborodov 2003; Nayakshin \& Sunyaev 2003) that also our Galaxy centre contained a similar gaseous disc at some stage of its history. Some form of an inactive disc or a dusty torus may still exist as a remnant of past activity. If such a disc existed in the Galaxy centre, it could capture passing stars from unbound orbits, or it could directly supply the medium where new stars form and evolve while sinking to the black hole. This mechanism has been explored under conditions relevant for active galactic nuclei and quasars (Collin \& Zahn 1999; Goodman $\&$ Tan 2004) and for Sgr $A^{\star}$ (Genzel et al. 2003). Likewise it has been speculated that star-disc collisions may contribute to the featureless X-ray variability by creating bright spots on the disc surface and fountains of ejected material above it (Zentsova 1983; Karas \& Vokrouhlický 1994; Vilkoviskij \& Czerny 2002). Cuadra et al. (2003) and Nayakshin et al. (2003, 2004 b) have pointed out that a similar mechanism may be responsible for X-ray flares, the occurrence of which was first reported by Baganoff et al. (2001). A sufficiently long observation could reveal periodicities of the stellar motion connected to precession of the orbit, and from them one would be able to infer the angular momentum of the central black hole (Semerák et al. 1999; Aschenbach et al. 2004).

We apply the model of star-disc collisions to the orbital evolution of the star S2, assuming a dissipative perturbation of its motion by crossing an inactive gaseous slab. We employ a similar formulation of the model as described by Šubr \& Karas (1999) and Karas \& Šubr (2001). The relative mass of the disc, $\epsilon \equiv M_{\mathrm{d}} / M_{\bullet}$, is a free parameter for us. We adopt a plausible and quite conservative value of $\epsilon$ in the range $10^{-3} \lesssim \epsilon \lesssim 10^{-2}$ (see also Nayakshin \& Cuadra 2004a; Šubr et al. 2004). Simulations are initialized at $r_{\mathrm{i}} \gtrsim 3 \times 10^{4} R_{\mathrm{g}}$ (i.e. above the present S2 distance; $R_{\mathrm{g}} \equiv G M_{\bullet} / c^{2} \approx 1.9 \times 10^{-7} \mathrm{pc}$ ) and we follow the sinking satellite as it proceeds to the centre. The gas-assisted drag tends to circularize the orbit and align it with the disc plane, while the Kozai (1962) mechanism drives eccentricity to a large value, characteristic of the present stage of S2. We employ this mechanism to explain the origin of the large orbital eccentricity and small pericentre. The assumed mass of gas distributed in the disc and the resulting perturbation of the predicted orbits are small enough to be consistent with Keplerian motion over one (or several) revolutions, which is the time span accessible to direct observational checks (see Mouawad et al. 2005, for an upper estimate of the mass outside the black hole). Clearly visible departures from perfectly elliptical orbits are expected on a substantially longer time-scale, $\approx 10^{3}$ orbits.

\section{The orbit evolution}

Let the gravitational field of the central black hole be described in the Newtonian approximation and the disc be planar and geometrically thin. Passages of a star through the disc last only a small fraction of the orbital period at the corresponding radius, and so they can be considered as instantaneous events at which the passing body exchanges momentum with the material lying along its trajectory. Repetitive events cause the orbit to decay. Outside the disc plane the star moves freely and its orbit is found numerically by integration in the combined gravitational field of the central mass $M_{\bullet}$ and the disc mass $M_{\mathrm{d}}$. This requires one to specify the surface density distribution as a function of radius in the disc and we assumed two cases for simplicity. First we employ a homogeneous slab $\Sigma_{\mathrm{d}}(R)=$ const. Its gravitational field can be then expressed analytically (e.g., Lass \& Blitzer 1983; Pierens \& Huré 2004), which helps to speed up computations. Another variant, $\Sigma_{\mathrm{d}}(R) \propto \sqrt{R\left(R_{\text {out }}-R\right)}$, was used to mimic the surface density and the gravitational field of an annulus ( $\left.R_{\text {in }} \leq R \leq R_{\text {out }}\right)$. Given the value of $M_{\mathrm{d}}$, the non-sphericity of the gravitational potential will be smaller and its impact on stellar orbits weaker if the disc matter is concentrated more towards the centre. However, we checked that a very similar effect to that described here is obtained even with the standard Shakura-Sunyaev density profile, i.e. decreasing with radius as $\Sigma_{\mathrm{d}}(R) \propto R^{-3 / 5}$. Most likely the actual density distribution will be somewhat different because the disc will be modified by self-gravity, stellar transitions and other effects ${ }^{1}$.

\footnotetext{
1 By the effects of self-gravity we understand the gravitational influence of the disc on the stars. As mentioned above, we do not modify the internal structure of the disc itself although this may also be interesting to examine because self-gravity is expected to cause a clumpy structure of the disc, thereby reducing the efficiency of star-disc collisions.
} 
The assumptions about the form of the disc are necessary for definiteness of examples, but they do not affect the nature of our results. If the trajectory is oriented in such a way that it crosses the disc repetitively, we observe the gradual dissipation of orbital energy and of angular momentum. As time proceeds, the orbit becomes circular and declined in the disc plane, but this overall trend is overlayed with oscillations of eccentricity and inclination. Occasional jumps toward high eccentricity occur due to self-gravity of the disc, acting as a perturbation in our model.

Kozai's method was developed to describe the evolution of mean orbital elements of a small body disturbed by the presence of another mass in the system. Our aim here is to consider a restricted problem in which the origin of perturbation is an accretion disc and the star represents a test body (a hierarchical triple system; see Lidov \& Ziglin 1975; Thomas \& Morbidelli 1996; Kinoshita \& Nagai 1999). Kozai's resonance is a particular type of secular resonance occurring when the pericentre argument precession vanishes, $\dot{\omega}=0$ for the test body. In order to disregard various short-time effects acting on dynamical time-scale, the method invokes averaging technique which is based on canonical transformation (Arnold 1989; Brower \& Clemence 1961; Morbidelli 2002). Although this formalism was suited and intensely applied to the problem of motion in the Solar system, it has met numerous applications elsewhere. For example, Holman et al. (1997) proposed that large eccentricities of the orbits of some extrasolar planets could be understood in terms of Kozai oscillations, which are caused by the disturbing influence of the secondary star. A similar approach was employed in the context of stellar dynamics around massive black holes in galactic nuclei (Sridhar \& Touma 1999) and in globular clusters (Wen 2003). Blaes et al. (2002) proposed that oscillations of eccentricity could be important for determining the rate of binary black hole merging and gravitational wave emission expected from galactic centres.

An important point to notice is the presence of dissipation due to stars crossing the disc. The star experiences orbital decay due to repetitive transitions changing the trajectory and pushing it across the separatrix. We consider two effects which both depend on the density of the gaseous environment: the energy dissipation is roughly proportional to the density of the medium and likewise the gravitational perturbation increases its impact proportionally to the mass contained in the disc. These two processes are therefore mutually connected. As far as star-disc collisions are concerned, relevance of the Kozai-type mechanism coupled with dissipation was originally discussed by Vokrouhlický \& Karas (1998b) and we refer the reader to their paper for further details. Here we briefly summarize the adopted approach.

The origin of the disturbing gravitational influence is selfgravity of the accretion disc, hence the perturbation potential is already assumed axially symmetric. Averaging is performed over the star ellipse. We denote the dominant potential of the central source as $V_{\mathrm{c}}(r)=-G M_{\bullet} / r$ (the central black hole) and the disturbing component $V_{\mathrm{d}}(R, z)$ (the disc). Test-particle motion in the referential background field is described by an unperturbed Hamiltonian, which is simply $\mathcal{H}_{\mathrm{c}}=-\frac{1}{2} G M_{\bullet} a^{-1}$ in terms of semi-axis $a$. Eccentricity and inclination are related by $^{2}$

$\eta \cos i=c, \quad$ with $\quad \eta \equiv \sqrt{1-e^{2}}$

and $c$ secularly constant (Kozai's integral). Along $c=$ const. orbital oscillations take place on substantially longer intervals than the dynamical period at corresponding distances, defining the medium time-scale $t_{\mathrm{s}}$ of the problem. A fairly accurate estimation of $t_{\mathrm{s}}$ can be obtained by replacing our disc with a narrow ring of radius $R_{\mathrm{d}}$ and the same mass $M_{\mathrm{d}}$ (e.g. Kiseleva et al. 1998),

$$
\begin{aligned}
t_{\mathrm{S}} & =\frac{2}{3 \pi} \epsilon^{-1}\left(\frac{R_{\mathrm{d}}}{a}\right)^{3} t_{\mathrm{p}} \\
& =556\left(\frac{\epsilon}{10^{-3}}\right)^{-1}\left(\frac{M_{\bullet}}{3.5 \times 10^{6} M_{\odot}}\right)\left(\frac{R_{\mathrm{d}}}{a}\right)^{3}\left(\frac{a}{10^{4} R_{\mathrm{g}}}\right)^{3 / 2} \mathrm{yr},
\end{aligned}
$$

where $t_{\mathrm{p}}(a)$ is the Keplerian orbital period and $R_{\mathrm{d}} \rightarrow R_{\text {out }}$ characterizes the size of the disc. More precisely, $c$ as well as $a$ would have been conserved if the energy dissipation had been negligible. However, the orbital energy is gradually dissipated in our system and this ensures that the star reaches the separatrix and passes through it, i.e., the orbital elements change their values and they eventually acquire a suitable combination. The overall orbital decay introduces the third time-scale, $t_{c}$, and we assume that it is the longest time-scale relevant for our problem (this assumption imposes an upper limit on density of the disc but one can check that the constraint is safely met under astrophysically realistic conditions). The total duration is obtained by integrating the inverse rate of orbital decay from the outer edge of the disc down to (say) tidal radius,

$t_{\mathrm{c}} \propto \int_{R_{\text {out }}}^{R_{\mathrm{T}}} \dot{a}^{-1} \mathrm{~d} a$,

where $\dot{a}<0$ depends on the surface density profile. Its magnitude is roughly $\propto \Sigma(R)$, but details are model dependent (Syer et al. 1991; Zurek et al. 1994). Following the arguments in Karas \& Šubr (2001) we estimate

$$
\begin{aligned}
t_{\mathrm{c}} \approx & 3 \times 10^{7} \Sigma_{3}\left(\frac{\epsilon}{10^{-3}}\right)^{-1}\left(\frac{M \cdot}{3.5 \times 10^{6} M_{\odot}}\right)^{2} \\
& \times\left(\frac{R_{\text {out }}}{10^{4} R_{\mathrm{g}}}\right)^{2}\left(\frac{a}{10^{4} R_{\mathrm{g}}}\right)^{3 / 2} \mathrm{yr} .
\end{aligned}
$$

Here we introduced $\Sigma_{3} \equiv \Sigma_{*} /\left(0.3 \Sigma_{\odot}\right)$ with $\Sigma_{*}=M_{*} / S_{*}$ and $S_{*}=\pi R_{*}^{2}$ (column density, mass, cross-sectional area and radius of the star, and analogically for the sun). $\Sigma_{3} \approx 1$ for $S 2$, i.e. a fiducial value for young, massive stars. Clearly the pe$\operatorname{riod} t_{\mathrm{c}}$ is inversely proportional to $R_{*}^{2}$ and found of the order of $t_{\mathrm{c}} \approx 10^{2} \mathrm{Myr}$ for typical values of the model parameters.

As mentioned above, the main difference between the direct numerical integration of stellar orbits and the analytical approach of the averaging technique lies in the way how the rapidly changing (fast) variables are treated. The mean anomaly plays the role of a fast variable in our problem,

\footnotetext{
${ }^{2}$ In our notation, $i=0^{\circ}$ corresponds to an orbit co-rotating with the disc, while $i=180^{\circ}$ is for counter-rotating one.
} 

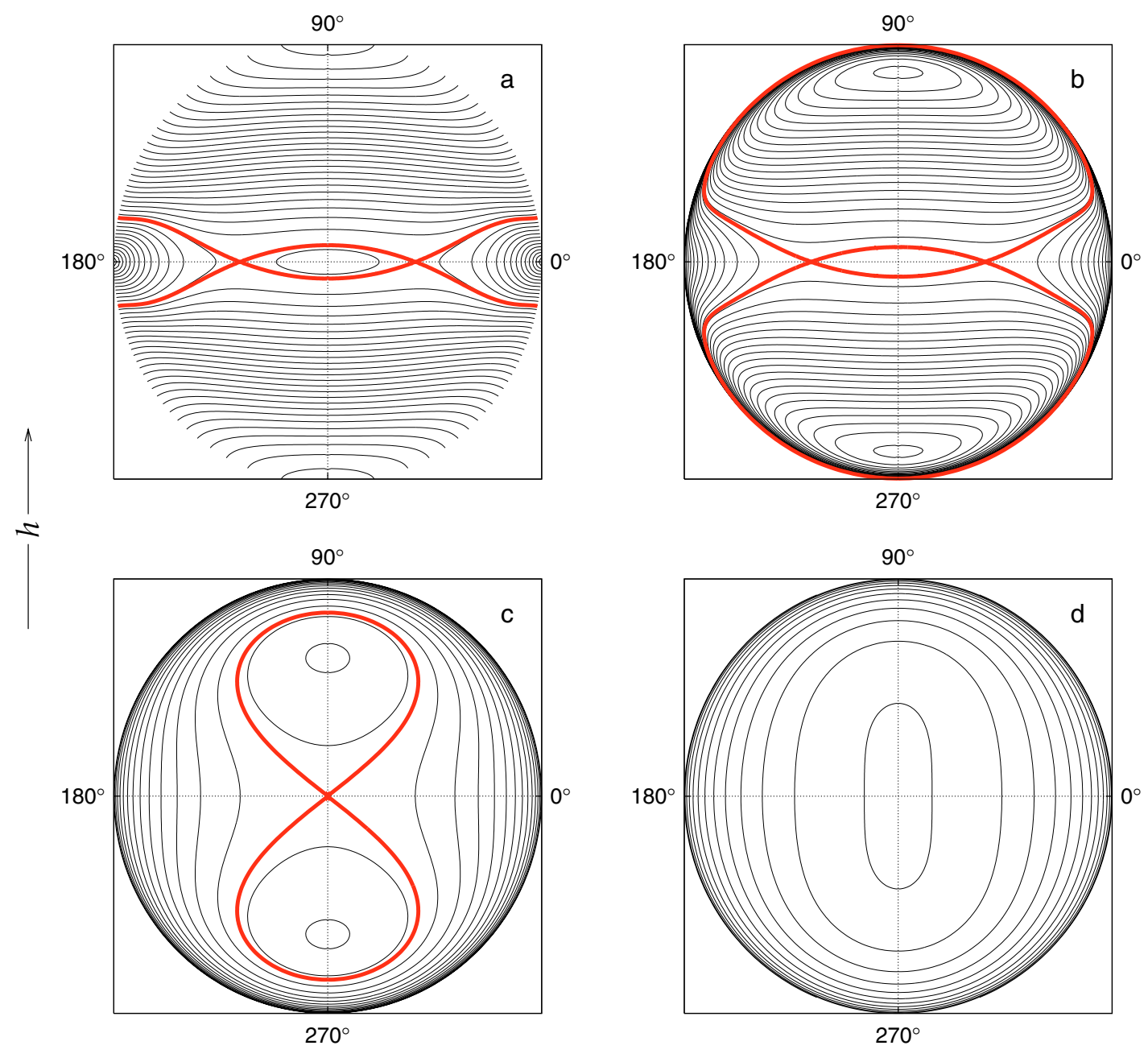

$k$

Fig. 1. Contour lines of the disturbing function $\bar{V}_{1}(\rho, \omega)$ (shown with equidistant steps between contour levels). The polar plot is shown with zero eccentricity at the origin $\rho=0(e=0)$ and polar angle $\omega$ indicated on margins. In the case of negligible dissipation the orbital evolution proceeds along contours. In different regions of the graph $\omega$ either circulates over the full circle or it librates within a limited interval. A separatrix (thick line) forms a border between these regions. Four topologically different cases can be distinguished by the structure of contours, shape of the separatrix and the corresponding value of Kozai constant $c$ : a) $c=0(e<1)$; b) $c=0.2(e<0.98)$; c) $c=0.8(e<0.60)$; d) $c=0.9(e<0.44)$. These define the range of orbital oscillations of eccentricity and inclination according to Eq. (1). Orbital energy has been set identical and equal to $-G M_{\bullet} /(2 a)$ with semi-axis $a=2.4 \times 10^{4} R_{\mathrm{g}}$ in all panels. While on the medium time-scale the orbits remain attached to underlying contours, allowing us to estimate the range of variations of eccentricity, on the long time-scale the orbits are driven across contours. This gradual diffusion leads to topology changes at some moments. In this figure we considered uniform surface density of the disc.

therefore, the aim is to find a canonical transformation under which the transformed Hamiltonian becomes independent of this quantity. The transformation depends on the form of the disturbing potential $V_{\mathrm{d}}(R, z)$, and so it cannot be given explicitly for an arbitrary profile of density in the disc. One can however introduce a formal development with respect to the small parameter $\epsilon \ll 1$. After transformation and expansion to the first order we find

$V_{\mathrm{d}} \rightarrow \bar{V}_{\mathrm{d}}=\bar{V}_{1}+O(\epsilon)$

The first term of the series can be written explicitly,

$\bar{V}_{1}=\frac{1}{2 \pi \eta} \int_{0}^{2 \pi}\left(\frac{r}{a}\right)^{2} V_{\mathrm{d}}(R, z) \mathrm{d} v$ where integration is performed over the true anomaly $v$ of the star for one revolution around the black hole. At this level of approximation the mean semimajor axis stays constant and differs from the osculating semimajor axis by short-period terms only (we therefore do not distinguish them in our notation). The problem is reduced to the evolution of the mean eccentricity $e$ and pericentre argument $\omega$, which are further constrained by $\bar{V}_{1}(e, \omega ; a, c)=$ const. When evaluating this condition, $a$ and $c$ are parameters.

Levels of $\bar{V}_{1}=$ const are shown in Fig. 1. We use nonsingular Poincaré coordinates $k(e, \omega)$ and $h(e, \omega)$, defined by $h^{2}+k^{2}=2(1-\eta)$ and $h / k=\tan \omega$. In order to draw these contours we construct a polar graph in $(\rho, \omega)$ with the azimuthal angle $0 \leq \omega<2 \pi$, polar radius $\rho \equiv \sqrt{h^{2}+k^{2}}$ and the origin 
$\rho=0$ coinciding with the circular orbit (the mean eccentricity increases with polar radius, $e \propto \rho$ near origin). The disc surface density was assumed constant.

Rigorous proof of convergence of the expansion (5) and an estimation of accuracy of the first term $\bar{V}_{1}$ in the series is a difficult task which cannot be assessed for a general perturbation potential. However, a partial check can be achieved by comparing the analytical result with numerical integration of exemplary trajectories (we will show such a comparison in the next section). Typically, results based on a truncated part of the series are valid over a limited time span and they fail to predict the system evolution for infinite duration.

We conclude this section by summarizing the main points in our perspective of how Kozai's phenomenon could help to solve the problem of S-stars in Sgr $\mathrm{A}^{\star}$ :

(i) Axially symmetric (non-spherical) perturbation of the central gravitational field is the essential element of the proposed scheme. We attribute its origin to self-gravity of the gaseous environment with the geometry of the disc.

(ii) Stars of the nuclear cluster follow nearly Keplerian orbits around the central black hole. Free motion in the central field is disturbed by the disc gravity.

(iii) Star-disc collisions lead to dissipation and gradual sinking toward the black hole, the decay rate being faster for counter-rotating and highly eccentric trajectories. Eccentricity of highly inclined orbits is pumped to large values via Kozai's mechanism. Consequently, the pericentre distance shrinks.

\section{Results}

Examples of integration of the evolving trajectory are shown in Fig. 2, where the orbital oscillations and the progressive sinking of the star to the centre are evident. We introduce rescaled time $\tau$ as a convenient measure, related to time $t$ in physical units by

$t=\Sigma_{3}\left(\frac{M \bullet}{3.5 \times 10^{6} M_{\odot}}\right)^{2} \tau$.

The characteristic time-scale $t_{\mathrm{s}}$ of the oscillations depends on $M_{\mathrm{d}} / M_{\bullet}$. ratio and is generally much longer that the dynamical time at the corresponding radius ( $t_{\mathrm{s}}$ is of the order of thousands of orbital revolutions in our case). On the other hand, the rate of orbital decay $t_{\mathrm{c}}$ is proportional to $\Sigma_{*}$ as indicated by Eq. (4), and so our results can be readily scaled to different stellar types. Furthermore, scaling can be performed to different values of $M_{\bullet}$ provided that the ratio $M_{\mathrm{d}} / M_{\bullet}$ is retained, as well as the disc dimensions and the orbital semimajor axis in units of $R_{\mathrm{g}}$.

Now we set the inner edge of the disc at $R_{\text {in }}=6 R_{\mathrm{g}}$ and the outer edge $R_{\text {out }}=5 \times 10^{4} R_{\mathrm{g}}$. The disc mass is $M_{\mathrm{d}}=10^{-3} M_{\bullet}$, which for $M_{\bullet}=3.5 \times 10^{6} M_{\odot}$ gives $\Sigma_{\mathrm{d}}=2.9 \times 10^{3} \mathrm{~g} \mathrm{~cm}^{-2}$ for surface density. In this case, Fig. 2 demonstrates how eccentricity is pumped to large values and, consequently, the orbital decay is accelerated. The semimajor axis decreases by a factor of 5 in several million years, even for a relatively moderate choice of the disc mass and density. The effect is thus potentially important, as it is able to bring stars near the black hole and put them on eccentric orbits. In these computations we employed a fourth-order Runge-Kutta integrator with adaptive stepsize. In order to reduce the duration of runs we pre-generated the field components in a Cartesian grid covering a rectangular region of $1 \mathrm{pc} \times 1 \mathrm{pc}$. In some regions, close to the disc, the field lines are highly distorted. Therefore, we used additional sub-grids that provide fine resolution where needed. Field values in between mesh points were obtained by bilinear interpolation. As detailed below, we can be fairly confident of sufficient accuracy of the numerical computation because the resulting trajectories agree with expectations based on analytical arguments of the perturbation method.

The orbital oscillations, which we observe in graphs, call for some kind of probabilistic interpretation of the orbital parameters. This can be inferred from non-uniform distribution of dots which represent fluctuations of $e(\tau)$ and $i(\tau)$ in Fig. 2 . Each dot represents the position in the phase space at a corresponding moment of time, and the density of dots determines the probability that the star occurs with given values of parameters $^{3}$. From Fig. 2 and especially from the detail in Fig. 3 we see that the oscillations of eccentricity and inclination are bound together by Kozai's quasi-integral $c \equiv \sqrt{1-e^{2}} \cos i=$ const.

Another representation of the orbital oscillations is given in Fig. 4 where we plot the time evolution of probability $p\left(\tau ; e_{\mathrm{c}}\right)$ that eccentricity has exceeded a given threshold $e_{\mathrm{c}}$. This graph demonstrates how a sinking star can, indeed, be set on a highly elliptic trajectory at certain stages, while gradually approaching the black hole. Figure 4 helps to answer the question of what fraction of time the orbit actually spends at the stage of high eccentricity. For example, by integrating $\int p\left(\tau ; e_{\mathrm{c}}=0.8\right) \mathrm{d} \tau$ over the first $6 \mathrm{Myr}$ we find the star spends approximately half its time with an eccentricity larger than 0.8 . Then the orbit becomes progressively circularized and the star descends below $10^{4} R_{\mathrm{g}}$.

Contour levels of the disturbing potential are shown in Fig. 5. We construct this graph using the same procedure as described for Fig. 1. Two panels correspond to slightly different moments; notice that there is a small change of the contour line structure because of continuous dissipation. In these plots we also include the exact (numerically integrated) trajectory. Clearly the adopted approximation is sufficiently accurate: the numerical trajectory follows its underlying contour at each corresponding panel, as it should. An adiabatic diffusion across $\bar{V}_{1}=$ const. lines is slow and it cannot be resolved in this figure, where $\omega$ circulates over the whole interval $\langle 0,2 \pi\rangle$ on the timescale of the order of several hundred orbital periods. However, there is an evident difference between the two cases: in the left panel the trajectory oscillates near the origin keeping small eccentricity, while on the right it has crossed the separatrix. As a result of this change, the corresponding orbital energy is also slightly different, whereas the mean eccentricity starts to vary over substantially larger intervals. This also explains how the

\footnotetext{
${ }^{3}$ To avoid fake structures in the graph, dots are plotted with random time steps that are distributed uniformly over $\left\langle 0, t_{\max }\right\rangle$, where $t_{\max }$ is an interval longer than the orbital time and shorter than characteristic time $t_{\mathrm{s}}$ of orbital oscillations.
} 

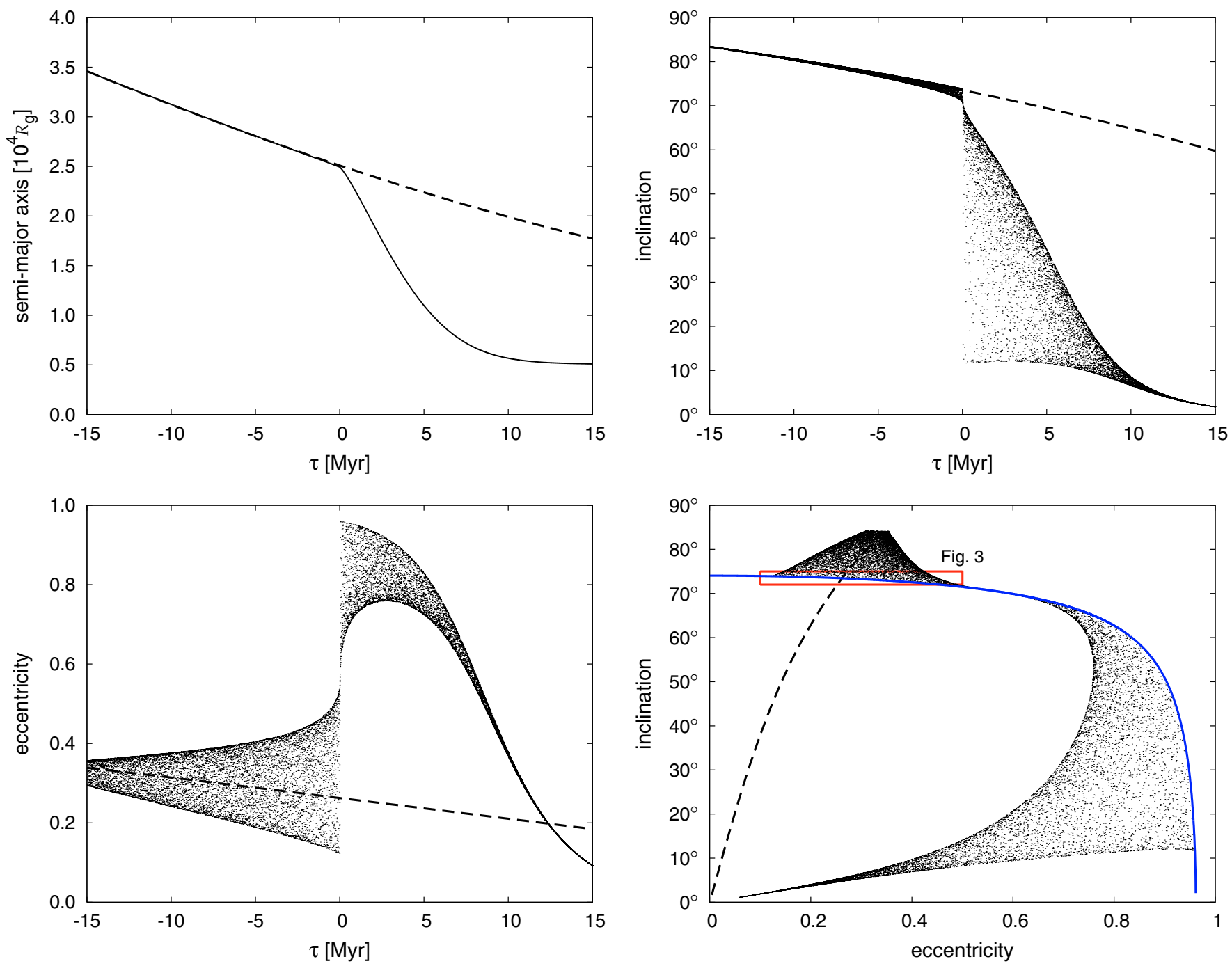

Fig. 2. Time evolution of a representative trajectory, which evolves according to the mutual interplay of gravitational and dissipative interaction with an accretion disc. Mean orbital elements are shown in different panels. The semi-axis $a(\tau)$ (solid line) monotonously decays as the star sinks to the centre. Inclination $i(\tau)$ and eccentricity $e(\tau)$ (plotted with points) oscillate with rather large amplitudes. We also show a reference orbit (dashed line), which was computed by neglecting the gravitational field of the disc. The oscillations and the Kozai resonance are due to the disc gravity; hence they affect only the exact trajectory and not the referential one. The orbital decay is due to dissipation, hence it affects both solutions. Both cases start with the same initial values: $a_{\mathrm{i}}=3.5 \times 10^{4} R_{\mathrm{g}}, e_{\mathrm{i}}=0.35$ and $i_{\mathrm{i}}=84^{\circ}$. Zero time has been set at the moment when the orbital parameters of the exact trajectory change their course abruptly, attaining the values consistent with those reported for the S2 star. We also show the graph of inclination vs. eccentricity (bottom right) with an elliptical arc segment corresponding to Kozai's $c=0.275$. The transition to a different regime of Kozai's oscillations occurs along this segment and a detailed subplot of the indicated rectangular area is enlarged in Fig. 3.

averaged model can be used to estimate the maximum eccentricity attained by the star.

In the above example the Kozai oscillations were only used to set the S2 orbit into a highly eccentric state. Indeed, it is evident from the orbit evolution at $\tau>0$ that its decay is considerably accelerated by this process. This is due to the fact that a star on a highly eccentric orbit passes through the disc with large relative velocity. The same argument suggests that counter-rotating stars suffer more from the orbital dissipation than co-rotating ones. Moreover, the efficiency of dissipation due to large inclination is strengthened because the separatrix prevents the overturning of counter-rotating orbits to co-rotation.
Hence, we found it convenient to explore the possibility that the S-stars have been formed on nearly circular orbits farther form the centre. Then, due to the simultaneous energy dissipation and the influence of the perturbing gravitational field the stars undergo orbital decay. Sample trajectories in Fig. 6 were integrated assuming $R_{\text {out }}=2 \times 10^{5} R_{\mathrm{g}}$ and $M_{\mathrm{d}} / M_{\bullet}=0.01$. Two different lines represent the orbital decay of a star interacting with different $\Sigma_{\mathrm{d}}(R)$ profiles starting with identical initial values of orbital elements.

The region of efficient star formation is usually placed at $r \approx 10^{6} R_{\mathrm{g}}$, however, the minimum radius is not known very precisely. Collin \& Zahn (1999) argue, using the model of a marginally unstable self-gravitating accretion disc, that the star 


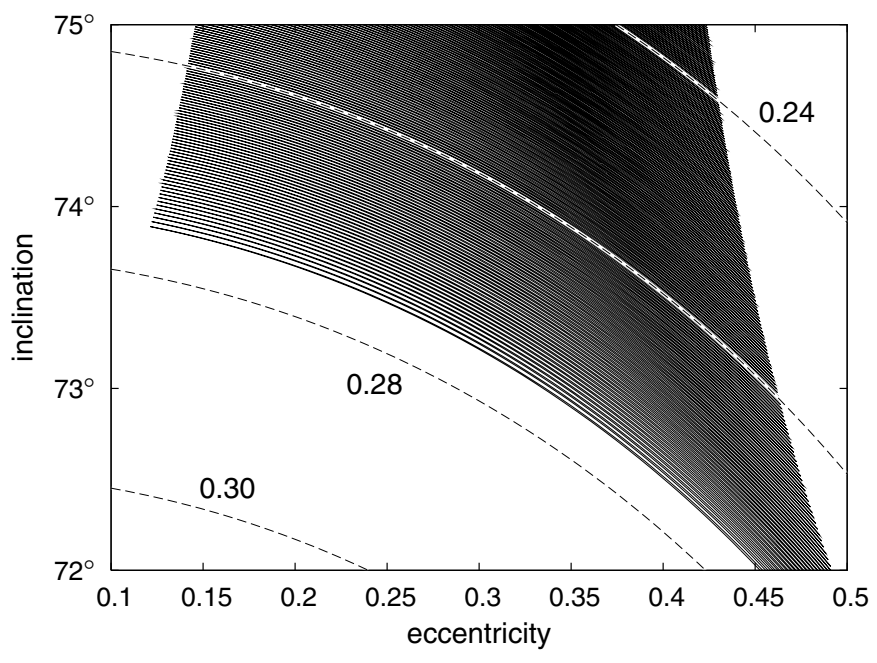

Fig. 3. Detail of the trajectory from Fig. 2, showing that the orbit (solid line) oscillates almost precisely along the lines of Kozai's integral (several contours are plotted with dashed lines and the corresponding values of $c=$ const. are given). This indicates that the disc mass is small but non-zero, and its gravity provides a perturbation of the central field of the black hole. The orbit diffuses across the $c$-curves due to orbital decay by dissipation.

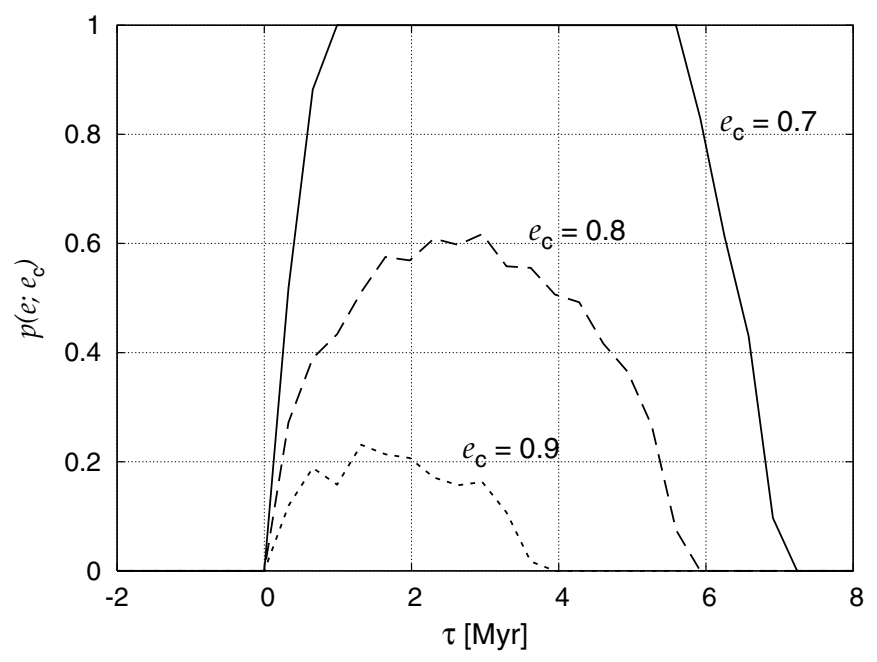

Fig. 4. The probability $p\left(\tau ; e_{\mathrm{c}}\right)$ of attaining eccentricity higher than $e_{\mathrm{c}}$ for the same example orbit as in Fig. 2. Three curves are shown for different values of $e_{\mathrm{c}}$. The eccentricity reaches large values during the period starting at $\tau=0$ and lasting about $6 \mathrm{Myr}$, and it stays above $e_{\mathrm{c}}=0.7$ for a considerable fraction of time.

forming process can take place at distances $\gtrsim 10^{5} R_{\mathrm{g}}$. Although their work was intended for quasars, it indicates that the mechanism discussed here could be relevant also for $\mathrm{Sgr}^{\star}$ if there is enough material dispersed around the black hole, or if it was there in the past. Star forming process occurring at roughly $10^{5} R_{\mathrm{g}}$ was also considered by Nayakshin \& Cuadra (2004a). Furthermore, it was suggested (Genzel et al. 2003) that young He I stars rotating in two coherent rings of radii $\gtrsim 10^{5} R_{\mathrm{g}}$ could have formed during a recent star forming episode triggered by a collision of different gaseous streams. Our two examples in Fig. 6 indicate that the exact form of the gas distribution play an essential role, both for the orbital decay and for oscillations.
Fine-tuning of the model parameters changes the quantitative results and allows us to decrease $M_{\mathrm{d}}$ but these details cannot be compared with present-day observations.

As explained above, jumps of mean eccentricity are caused by the Kozai process in an non-spherical (axially symmetric) perturbation of the central gravitational field. This we interpret as the influence of the disc, which simultaneously provides the dissipative environment in a self-consistent manner. The process of Kozai-driven resonance prefers rather special orientation of initial trajectories, namely, almost perpendicular inclination. We can therefore speculate that the current family of S-stars was born jointly and these were originally moving in a similar direction. They experienced the Kozai resonance and underwent the orbital decay ending in the present state of highly elliptic trajectories very near the black hole. Some of the nuclear stars in Sgr A* (Genzel et al. 2003; Levin \& Beloborodov 2003) form a disc-like structure rather than a spherical distribution (nuclear stars are actually located in two rings, almost perpendicular to each other; Genzel et al. 2003). We can thus consider such rings as current prototypes of a formerly existing ring in which S-stars were formed.

\section{Discussion}

In this paper we considered two complementary effects which both act as perturbations on otherwise free Keplerian motion, namely, gravity of the disc and the orbital decay due to crossing the disc slab. We computed a set of model trajectories of stars sinking towards a supermassive black hole in Sgr A*. We considered the region of gravitational dominance of the central black hole, however, we also took the gravity of the accretion disc and the corresponding dissipation into account. Stars do not strictly follow test-body (geodesic) motion in the central field, but instead they gradually sink to the centre and they experience occasional jumps of mean orbital elements. These second-order effects do not influence the motion on short timescales of the order of one or several revolutions, nevertheless they are essential for long time-scales. Although the existence of a fossil disc in the Galaxy centre is a matter of debate, the idea offers a natural explanation for various pieces of observational information, such as the occurrence of X-ray flares or the origin of young OB-type stars on highly elongated orbits. We therefore adopted the $\mathrm{S} 2$ orbital parameters in our simulations.

A more complete picture will require not only a detailed knowledge of the disc structure but also coupling with other effects, such as dynamical friction, gravitational radiation losses and the internal dissipation by tidal forces, which also contribute to the orbital decay, as well as the Lense-Thirring precession caused by rotation of the black hole. These and other mechanisms are beyond the scope of the present paper. Likewise, we neglected the effect of grazing encounters with other stars in the nuclear cluster. All these processes will have to be taken into account in order to disentangle their impact on the evolving orbit and to measure black hole parameters in the future. Our model predicts that stars spend a considerable fraction of total time at the stage of large eccentricity. This can be reconciled with the current evidence which strongly prefers high eccentricities in the central arcsecond region of $\operatorname{Sgr} A^{\star}$, 

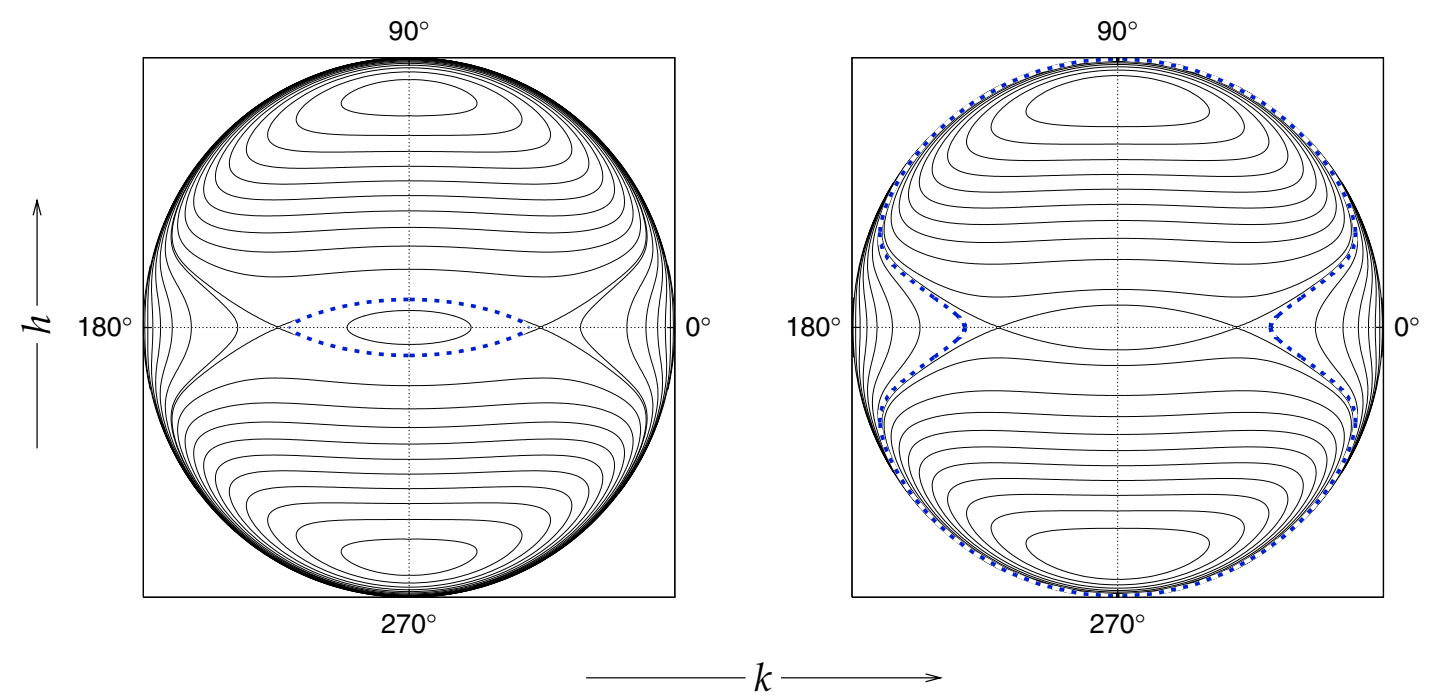

Fig. 5. The contour plot of disturbing potential $\bar{V}_{1}(\rho, \omega)=$ const shown at two moments of time. The exact (numerically integrated) trajectory is also plotted (by points). The left panel corresponds to the moment just before the numerical trajectory crosses the separatrix: $\tau=-0.15 \mathrm{Myr}$, $a=2.50 \times 10^{4} R_{\mathrm{g}}$. The corresponding value of Kozai's $c=0.2733$ and the maximum eccentricity $e_{\max }=0.962$. On the other hand, the right panel captures the orbit after crossing the separatrix: $\tau=0.15 \mathrm{Myr}, a=2.46 \times 10^{4} R_{\mathrm{g}}, c=0.2795, e_{\max }=0.960$.
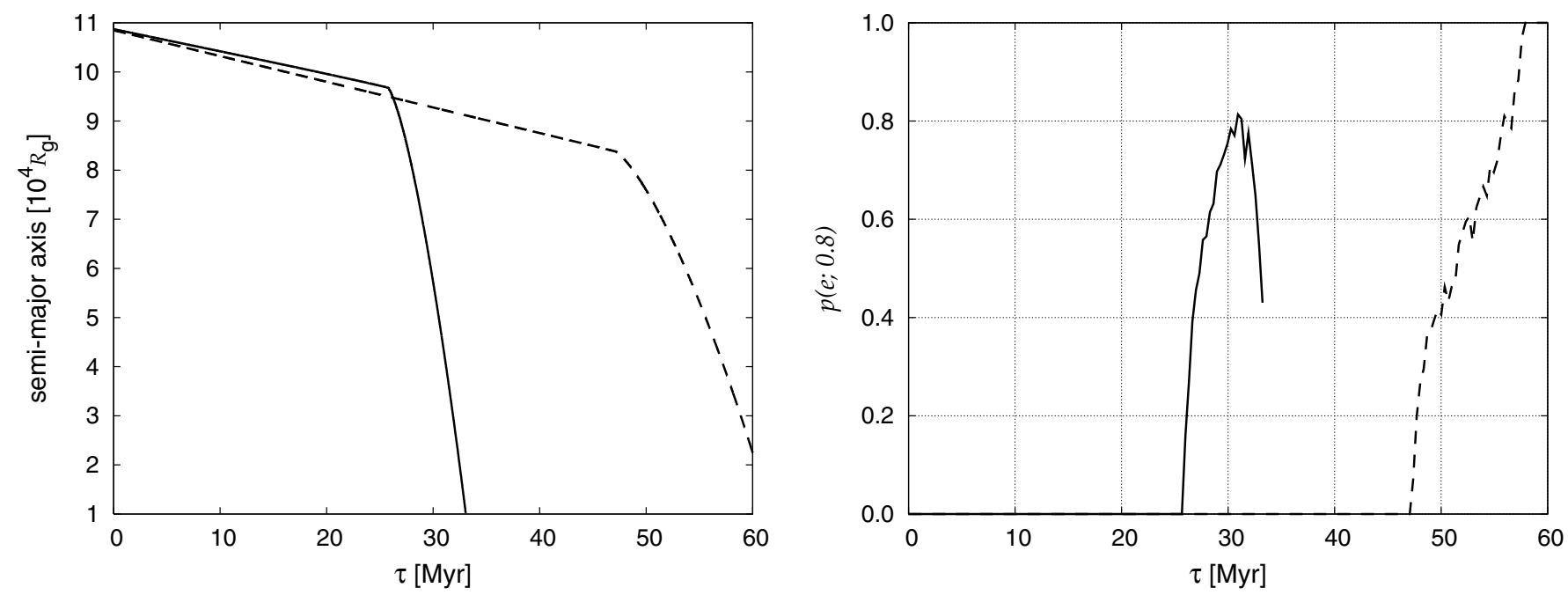

Fig. 6. Left: the orbital decay due to interaction with the disc. Two cases are shown corresponding to a homogeneous disc $\Sigma_{\mathrm{d}} \propto$ const. (solid line), and $\Sigma_{\mathrm{d}} \propto \sqrt{R\left(R_{\mathrm{out}}-R\right)}$ (dashed line) representing an annulus. The rate of gas-assisted orbital decay speeds up at the moment of crossing the separatrix (time $\tau=25 \mathrm{Myr}$ and $\tau=47 \mathrm{Myr}$, respectively). Right: the probability for these orbits to have eccentricity larger than 0.8 . Both trajectories start with identical initial values, $a_{\mathrm{i}}=1.1 \times 10^{5} R_{\mathrm{g}}, e_{\mathrm{i}}=0.15$ and $i_{\mathrm{i}}=105^{\circ}$, and at some stage they attain the values compatible with the S2-star parameters, i.e. $a \approx 2.5 \times 10^{4} R_{\mathrm{g}}$ and $e \approx 0.87$.

because highly elliptical orbits are preferentially brought to small radii.

The system of stars on energetically bound trajectories encircling a supermassive black hole is a unique configuration allowing one to probe the gravitational field of the black hole by tracking the stellar proper motion. The available accuracy has justified the approximation of free motion, assuming that the stars behave as test particles that are insensitive to nongravitational forces. This is fully satisfactory on the time-scale of several revolutions, i.e., of the order of tens to hundreds of years. However, we showed that the effect of gaseous and/or dusty environment should not be neglected in the discussion of the long-term (Myr) evolution and that it can in fact help to reconcile the problem of the origin of the stellar population in the Galaxy centre. Although our approach involves various simplifications, the main message here is that one should take both effects into account simultaneously, i.e. the orbital dissipation in the gaseous environment of the disc and its gravitational field. In fact, the idea of combining gravitational (conservative) and hydrodynamical (dissipative) star-disc interactions can be considered as a model case for a more general kind of process introducing a non-spherical perturbation to the central gravitational field and, simultaneously, a dissipative mechanism for the orbital decay. 
Acknowledgements. We thank S. Collin for valuable discussions about star formation in black-hole accretion discs with self-gravity, and to K. Beckwith, C. Hopman, N. Mouawad and D. Vokrouhlický for comments. We also thank our referees, S. Nayakshin and J. Cuadra, for helpful criticism improving the paper. We gratefully acknowledge the financial support from the Czech Science Foundation under grants 205/02/P089 (L.Š.) and 205/03/0902 (V.K.), as well as Charles University in Prague (299/2004).

\section{References}

Alexander, T., \& Hopman, C. 2003, 560, L143

Alexander, T., \& Livio, M. 2001, 590, L29

Alexander, T., \& Livio, M. 2004, 606, L21

Armitage, P. J., Zurek, W. H., \& Davies, M. B. 1996, ApJ, 470, 237

Arnold, V. I. 1989, Mathematical Methods of Classical Mechanics (Berlin: Springer-Verlag)

Artymowicz, P. 1993, ApJ, 419, 166

Artymowicz, P., Lin, D. N. C., \& Wampler, E. J. 1993, ApJ, 409, 592

Aschenbach, B., Grosso, N., Porquet, D., \& Predehl, P. 2004, A\&A, 417,71

Baganoff, K., Bautz, W., Brandt, N., et al. 2001, Nature, 413, 45

Blaes, O., Lee, M. H., \& Socrates, A. 2002, ApJ, 578, 775

Brower, D., \& Clemence, G. 1961, Methods of Celestial Mechanics (New York: Academic Press)

Collin, S., \& Zahn, J.-P. 1999, A\&A, 344, 433

Cuadra, J., Nayakshin, S., \& Sunyaev, R. 2003, A\&A, 411, 405

Eckart, A., Moultaka, J., Viehmann, T., Straubmeier, C., \& Mouawad, N. 2004, ApJ, 602, 760

Genzel, R., Schödel, R., Ott, T., et al. 2003, ApJ, 594, 812

Ghez, A. M., Becklin, E. E., Duchêne, G., et al. 2003a, Astron. Nachr., 324,527

Ghez, A. M., Duchêne, G., Matthews K., et al. 2003b, ApJ, 586, L127

Goodman J., \& Tan, J. C. 2004, ApJ, 608, 108

Hameury, J.-M., King, A. R., Lasota, J.-P., \& Auvergne, M. 1994, A\&A, 292, 404

Hansen, B. M. S., \& Milosavljević, M. 2003, ApJ, 593, L80

Holman, M., Touma, J., \& Scott, T. 1997, Nature, 386, 254
Karas, V., \& Šubr, L. 2001, A\&A, 376, 686

Karas, V., \& Vokrouhlický, D. 1994, ApJ, 422, 208

Kinoshita, H., \& Nakai, H. 1999, Celest. Mech., 75, 125

Kiseleva, L. G., Eggleton, P. P., \& Mikkola, S. 1998, MNRAS, 300, 292

Kozai, Y. 1962, AJ, 67, 591

Lass, H., \& Blitzer, L. 1983, Celest. Mech. Dyn. Astron., 30, 225

Levin, Y., \& Beloborodov, A. M. 2003, ApJ, 590, L33

Lidov, M. L., \& Ziglin, S. L. 1976, Celest. Mech., 13, 471

McMillan, S. L. W., \& Portegies Zwart, S. F. 2003, ApJ, 596, 314

Milosavljević, M., \& Loeb, A. 2004, ApJ, 604, L45

Morbidelli, A. 2002, Modern Celestial Mechanics (London: Taylor \& Francis)

Morris, M. 1993, ApJ, 408, 496

Mouawad, N., Eckart, A., Pfalzner, S., et al. 2005, Astron. Nach., accepted [arXiv: astro-ph/0402338]

Nayakshin, S., \& Cuadra, J. 2004a, A\&A, submitted [arXiv:astro-ph/0409541]

Nayakshin, S., Cuadra, J., \& Sunyaev, R. 2004b, A\&A, 413, 173

Nayakshin, S., \& Sunyaev, R. 2003, MNRAS, 343, L15

Pierens, A., \& Huré, J.-M. 2004, ApJ, 605, 179

Rauch, K. 1995, MNRAS, 275, 628

Schödel, R., Ott, T., Genzel, R., et al. 2003, ApJ, 596, 1015

Schödel, R., Ott, T., Genzel, R., et al. 2002, Nature, 419, 694

Semerák, O., Karas, V., \& de Felice, F. 1999, PASJ, 51, 571

Sridhar, S., \& Touma, J. 1999, MNRAS, 303, 483

Šubr, L., \& Karas, V. 1999, A\&A, 352, 452

Šubr, L., Karas, V., \& Huré, J.-M. 2004, MNRAS, 354, 1177

Syer, D., Clarke, C. J., \& Rees, M. J. 1991, MNRAS, 250, 505

Thomas, F., \& Morbidelli, A. 1996, Celest. Mech. Dyn. Astron., 64, 209

Vilkoviskij, E. Y., \& Czerny, B. 2002, A\&A, 387, 804

Vokrouhlický, D., \& Karas, V. 1993, MNRAS, 265, 365

Vokrouhlický, D., \& Karas, V. 1998a, MNRAS, 293, L1

Vokrouhlický, D., \& Karas, V. 1998b, MNRAS, 298, 53

Wen, L. 2003, ApJ, 598, 419

Zentsova, A. S. 1983, Ap\&SS, 95, 11

Zurek, W. H., Siemiginowska, A., \& Colgate, S. A. 1994, ApJ, 434, 46 